\title{
RETINAL PROJECTIONS IN TYROSINASE-NEGATIVE ALBINO $\operatorname{CATS}^{1}$
}

\author{
DONNELL CREEL, ${ }^{2}$ ANITA E. HENDRICKSON, ${ }^{*}$ AND AUDIE G. LEVENTHAL $\ddagger$ \\ Veterans Administration Medical Center, Salt Lake City, Utah 84148; ${ }^{*}$ Department of Uphtr almology, University of \\ Washington School of Medicine, Seattle, Washington 98195; and $\ddagger$ Department of Anatomy, University of Utah School of \\ Medicine, Salt Lake City, Utah 84132
}

Received January 11, 1982; Revised February 25, 1982; Accepted February 26, 1982

\begin{abstract}
Retinal projections were examined in two tyrosinase-negative albino cats using autoradiographic techniques. Cats from this colony have pink eyes; their retinal pigment epithelium, ciliary body, and iris epithelium are completely devoid of melanin pigment. Test breeding for five generations indicates that these cats are true albinos (cc).

The most striking feature of the albino cats' visual pathways was a reduction in ipsilateral input which was more severe than that reported for Siamese cats. The only evidence of ipsilateral input to the laminated dorsal lateral geniculate nucleus of the albino was a small lateral normal segment and a small projection to the lateral portion of lamina C1. Ipsilateral projections to the medial interlaminar nucleus, retinal recipient zone of the pulvinar complex, ventral lateral geniculate nucleus, superior colliculus, and pretectum also were reduced. Ipsilateral projections to the suprachiasmatic nucleus appeared to be normal in the albino cats studied.

Our findings indicate that, compared to the normal cat as well as to the tyrosinase-positive Siamese cat, the tyrosinase-negative albino has reduced ipsilateral retinal projections. The albino cat is a model system analogous to tyrosinase-negative albinism found in mammals.
\end{abstract}

Visual system anomalies associated with forms of albinism in mammals have been reported for a number of years. Until recently, a complete form of albinism was not available in the domestic cat. The albino model that has been most studied is the Siamese cat. Guillery (1969) first described the abnormal retinogeniculate projection in Siamese cats, and in 1971, several publications appeared concerning the visual system of the Siamese cat (Creel, 1971a, b; Guillery and Kaas, 1971; Hubel and Wiesel, 1971; Kalil et al., 1971). Creel (1971a, b) pointed out that the Siamese cat possesses a mutant allele of the albino series at the $C$ locus $\left(c^{\mathrm{h}} c^{\mathrm{h}}\right)$ and suggested that the derangement of optic projections is associated with albinism throughout mammals. The abnormal retinogeniculostriate projections of Siamese cats have been described in detail (Guillery and Kaas, 1971; Hubel and Wiesel,

\footnotetext{
1 This work was supported by the Veterans Administration and Public Health Service Grants EY01208 (to A. E. H.) and EY03427 (to A. G. L.). The skilled technical assistance of DeVeta Moe and Lisa Hendrickson and the helpful advice of Paul Dice, DVM are gratefully acknowledged.

${ }^{2}$ To whom correspondence should be addressed at Neuropsychology Research 151A, Veterans Administration Medical Center, Salt Lake City, UT 84148.
}

1971; Guillery, 1974; Shatz, 1977; Cooper and Pettigrew, 1979). The picture that has emerged is that the ipsilateral retinal projection is reduced because many axons originating in the temporal retina which project ipsilaterally in normal cats project contralaterally in Siamese cats. While retinal projections in all Siamese cats appear abnormal, there is significant variability from one animal to the next in the extent of the chiasmatic abnormality (Stone et al., 1978; Cooper and Pettigrew, 1979) as well as in the way that the geniculocortical pathways compensate for the abnormal inputs from the contralateral temporal retina (Guillery and Kaas 1971; Hubel and Wiesel, 1971).

The Siamese cat is a form of partial albinism with definite retinal hypopigmentation. Retinal hypopigmentation is the principal prerequisite for misrouted central optic projections (Sanderson et al., 1974; Creel et al., 1974, 1978; Creel and Giolli, 1976; Wise and Lund, 1976; LaVail et al., 1978; Guillery et al., 1979). We have studied the retinal projections in tyrosinase-negative albino cats using autoradiography.

\section{Materials and Methods}

The albino cats were identified by the senior author (Creel and Todd, 1979). Five generations of these cats 
have been test bred. Inbreeding has produced only albino cats. Test breedings with Siamese cats indicate homology for the $C$ locus. Inheritance of this allele is autosomal recessive. All albino cats in this colony have pink eyes, nystagmus, and some form of strabismus. The auditory system of every cat in the colony has been tested using auditory brainstem responses for normal hearing thresholds. There has never been a deaf cat born among 20 cats across five generations.

The presence of the functional melanocyte activity necessary to produce melanin pigmentation can be detected by a chemical test that is used routinely to categorize types of human albinos (King and Witkop, 1976). When an anagen (growing) human hair bulb or cat vibrissa is plucked, a local concentration of melanocytes remains at the base of the bulb in both normally pigmented and albino mammals. Human hair bulbs or cat vibrissae can be tested for melanocyte activity by incubation in $80 \mathrm{mg}$ of L-tyrosine $/ 100 \mathrm{ml}$ of $0.1 \mathrm{M}$ phosphate buffer, pH 6.8 for $12 \mathrm{hr}$. See Witkop et al. (1978) for a review of the delineation of melanocytes in hair bulbs and tests for their chemical activity. Vibrissae from all 10 albino cats currently in the colony and 3 Siamese cats were tested for tyrosinase activity.

The vitreous of the left eye of an adult male albino cat was injected with $250 \mu \mathrm{Ci}$ of $\left[{ }^{3} \mathrm{H}\right]$ proline $(60 \mathrm{Ci} / \mathrm{mmol})$ in $20 \mu \mathrm{l}$ of saline and the vitreous of the right eye of an adult female albino cat received $250 \mu \mathrm{Ci}$ of $\left[{ }^{3} \mathrm{H}\right]$ leucine in 20 $\mu \mathrm{l}$ of saline. These animals were separated by three generations in the same pedigree. A normally pigmented adult female cat was injected with $250 \mu \mathrm{Ci}$ of $\left[{ }^{3} \mathrm{H}\right]$ proline in $20 \mu \mathrm{l}$ of saline into the right eye. Twenty-four to $40 \mathrm{hr}$ after injection, the animals were anesthetized deeply and perfused intravascularly with phosphate-buffered $4 \%$ paraformaldehyde. Their brains were sectioned coronally at $30 \mu \mathrm{m}$ using a freezing microtome, and every third section from the optic chiasm through the superior colliculus was mounted on subbed slides, coated with Kodak NTB-2 emulsion, and exposed at $4^{\circ} \mathrm{C}$ in the dark for 4 to 8 weeks. The slides were developed in Kodak D-19, fixed in Kodak Rapid Fix, and counterstained with cresyl violet (Hendrickson and Edwards, 1978).

The eyes were embedded in glycol methacrylate and sectioned serially at $4 \mu \mathrm{m}$. Sections were stained with Mallory trichrome or azure II-methylene blue; matching unstained sections also were prepared.

\section{Results}

Vibrissae from these albino cats showed the physical presence of melanocytes. Hair bulb incubation tests of the biochemical activity of melanocytes in vibrissae of all 10 albinos currently in the breeding colony indicated no tyrosinase activity. Melanocytes in the vibrissae of the 3 Siamese cats produced pigment when incubated in tyrosine at room temperature. The retinal pigment epithelium, ciliary body, and iris of our presumed albino cats were found to be completely devoid of melanin pigment in stained and unstained sections. Morphologically, the retinae appeared qualitatively normal with no obvious sign of photoreceptor atrophy.

Figures 1 to 4 are dark-field photographs of coronal sections through the optic chiasm, dorsal lateral geniculate nucleus (dLGN), pretectum, and superior colliculus. In these figures, the autoradiographic silver grains mark accumulations of radioactive protein in axons and synaptic terminals and appear light against the dark background. All figures are from the cat injected with $\left[{ }^{3} \mathrm{H}\right]$ leucine that survived $40 \mathrm{hr}$. The results for the second albino did not differ significantly from those illustrated in Figures 1 to 4.

Figure 1 shows the optic chiasm and illustrates the paucity of uncrossed (ipsilateral) optic tract fibers in

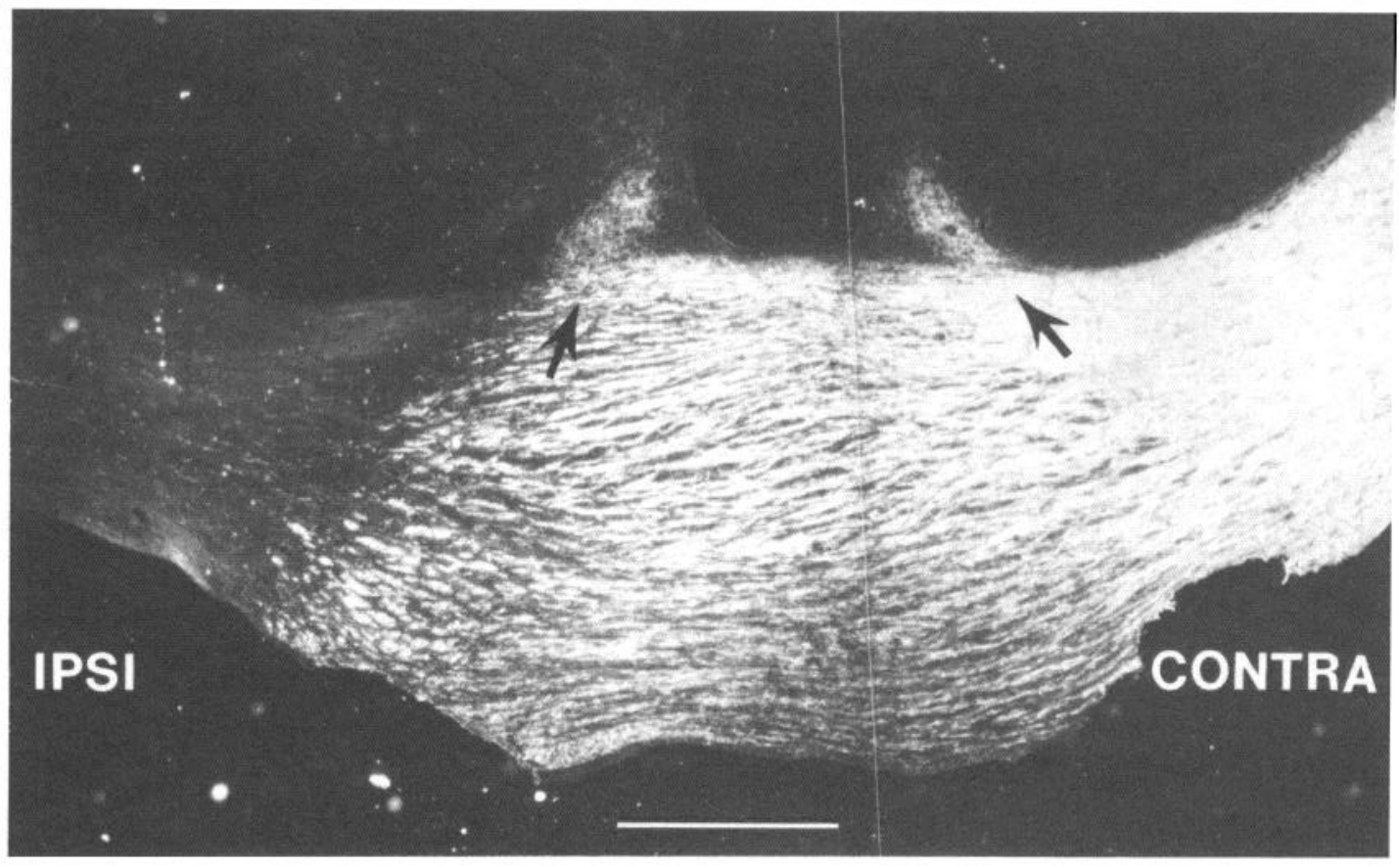

Figure 1. Dark-field autoradiograph of a coronal section of the optic chiasm of an albino cat after the injection of tritiated leucine into one eye. The arrows point toward fibers projecting into suprachiasmatic nuclei. Scale bar, $1 \mathrm{~mm}$. 
albino cats. On the other hand, the suprachiasmatic nuclei received nearly equal projections from the two eyes. Sample counts from the areas of the highest concentration of terminals indicated a ratio of approximately 1.4:1, comparing contralateral to ipsilateral suprachiasmatic projections. This was the only apparently normal ipsilateral input found in any central retinorecipient nucleus in these albino brains.

Figure 2 compares the ipsilateral $(A)$ and contralateral $(B)$ projections to the dLGN of a normally pigmented cat with the ipsilateral $(C)$ and contralateral $(D)$ projections of an albino cat. The normal cat demonstrated ipsilateral $(A)$ radioactivity across the extent of lamina $\mathrm{A} 1$, a well filled lamina $\mathrm{C} 1$, the medial interlaminar nucleus (MIN), and the retinal recipient zone (RRZ) of the pulvinar complex. The contralateral dLGN of the normal cat $(B)$ shows the appropriate areas corresponding to the regions receiving ipsilateral inputs to be vacant of labeling. The ipsilateral dLGN in the albino cat $(C)$ was devoid of radioactivity except for a small ovoid lateral normal segment and a small streak of lateral lamina $\mathrm{C} 1$. The contralateral dLGN of the albino cat $(D)$ was almost entirely filled with label except for a small patch corresponding to the location of the lateral normal segment. The location of the ovoid lateral normal segment of lamina A1 in both albino cats indicated that the part of the visual field represented was well beyond $20^{\circ}$ in the temporal retina (Sanderson, 1971). In both albinos, ipsilateral labeling of the ventral LGN was extremely weak, and the ipsilateral RRZ and MIN also contained very little evidence of radioactivity.

Figures 3 and 4 depict ipsilateral and contralateral retinal pathways in the pretectum and superior colliculus of the albino cat. There was very little radioactivity in the ipsilateral pretectum, while the ipsilateral superior colliculus contained a small medial patch of light label.

\section{Discussion}

The animals in this study should not be confused with "deaf white cats" which often are referred to as albinos. The white coat of a deaf white cat $(W)$ is produced by a spotting disorder caused by the failure of melanoblasts to migrate from the neural crest. The cat is all one white "spot" because it lacks pigment-producing cells. Thus, the hair bulbs of deaf white cats contain no melanocytes. Along with the failure of precursor pigment cells to migrate from the neural crest, there is often a failure of migration of the neural precursors to the inner ear in these cats, producing sensorineural deafness (Bergsma and Brown, 1971; Pujol et al., 1977). Geneticists consider the absence of pigment-producing cells to be a spotting disorder and albinism, per se, to be due to a chemical defect (Searle, 1968). The presence of melanocytes, the pigment-producing cell, can be detected in a human hair bulb or vibrissa of a cat by microscopic examination. The vibrissae of the albino cats in this study had melanocytes, but they were chemically tyrosinase-negative. The tyrosinase $(C)$ locus regulates the enzyme which facilitates
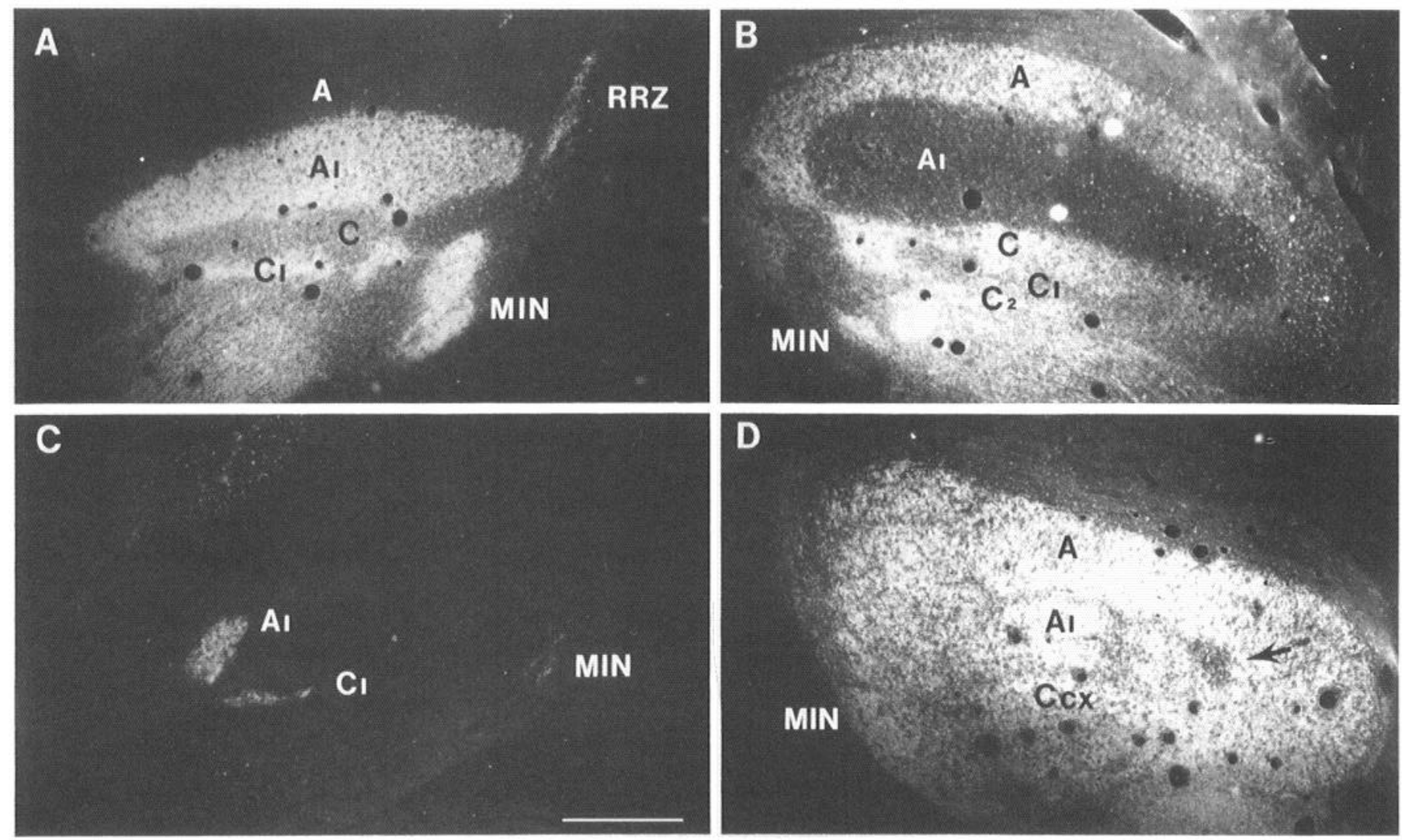

Figure 2. Dark-field autoradiographs of coronal sections through the ipsilateral $(A)$ and contralateral $(B)$ LGN of a normally pigmented cat and the ipsilateral $(C)$ and contralateral $(D)$ LGN of an albino cat. The arrow in contralateral section $D$ points to a pocket corresponding to the small lateral normal lamina $\mathrm{A} 1$ input seen in the ipsilateral section $C . A, A 1, C, C 1$, and $C 2$, laminae of the dorsal lateral geniculate nucleus; $C c x, \mathrm{C}$ laminae complex; $M I N$, medial interlaminar nucleus; $R R Z$, retinal recipient zone. Scale bar, $1 \mathrm{~mm}$. 

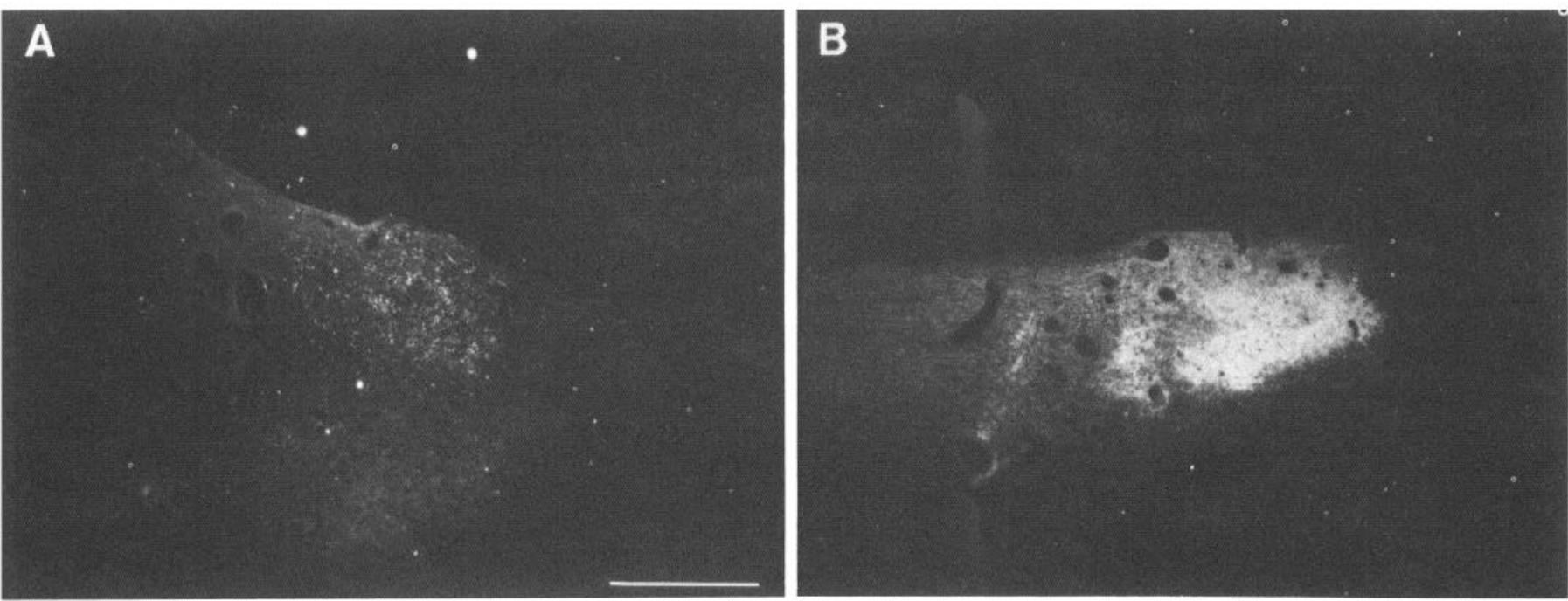

Figure 3. Dark-field autoradiographs of coronal sections through the ipsilateral $(A)$ and contralateral $(B)$ pretectum of an albino cat. Scale bar, $1 \mathrm{~mm}$.
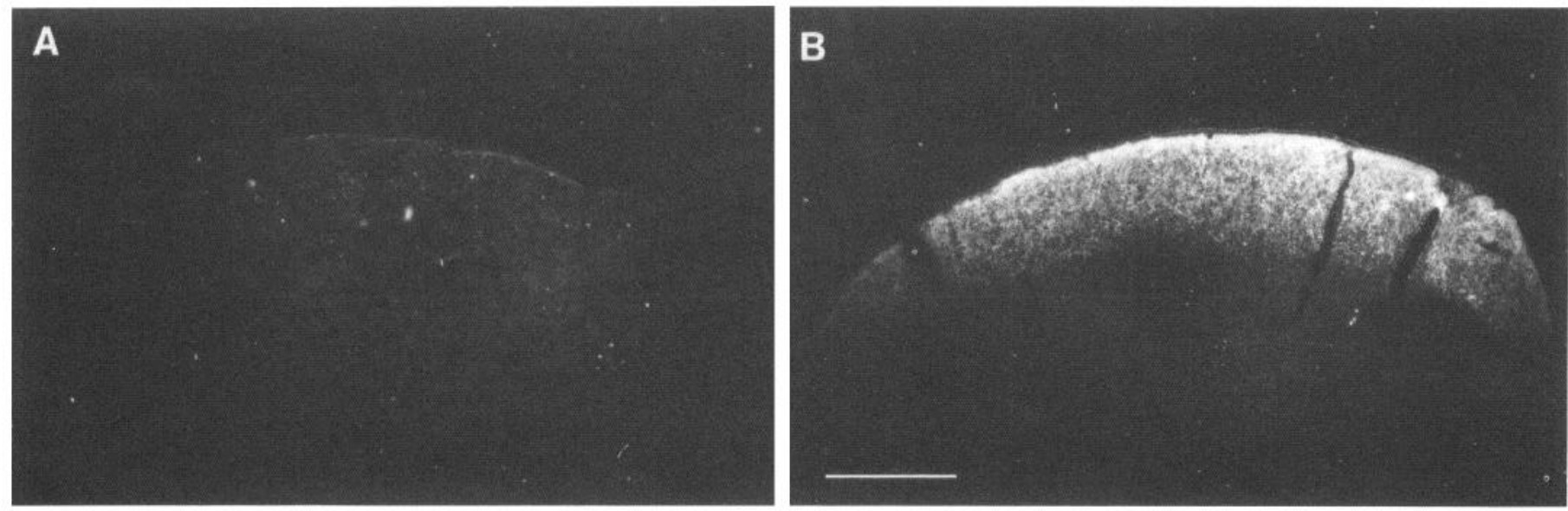

Figure 4. Dark-field autoradiographs of coronal sections through the ipsilateral $(A)$ and contralateral $(B)$ superior colliculus of an albino cat. Scale bar, $1 \mathrm{~mm}$.

the conversion of tyrosine to dopa and dopa to dopa quinone, thus initiating the chemical melanin pigmentation pathway (Creel, 1980; Prota, 1980). The most extreme form of albinism is completely tyrosinase-negative. The cats that we examined are completely tyrosinasenegative, i.e. incapable of producing melanin pigmentation.

Siamese cats have blue eyes; deaf white cats have blue eyes or heterochromia irides with one blue and one yellow eye. The eyes of all albino cats in this colony are pink and devoid of melanin pigment. The histology of the eyes of the cats studied verified that no melanin pigmentation was present in the iris, ciliary body, and retinal pigment epithelium. Based on the breeding studies, absence of melanin pigment in the eyes, tyrosinasenegative hair bulb incubation tests, and definite misrouting of the ipsilateral retinofugal projections, we conclude that these cats are true complete albinos.

Misrouting of the retinofugal projections is associated with metabolic forms of albinism, per se, and not with the absence of pigment cells due to a defect in migration of melanoblasts from the neural crest which produces an all white animal, such as the deaf white cat $(W)$. Cats that are white due to a deficiency of pigment cells have normal optic projections unless they are also homozygous for an albino gene, such as Siamese $\left(c^{\mathrm{h}} c^{\mathrm{h}}\right)$ genes (Thibos et al., 1980; Guillery et al., 1981).

All nondecussating retinofugal projections appear to be reduced more severely in tyrosinase-negative albino cats than in Siamese cats. Thus, there is a progressive reduction of input to most ipsilateral visual centers from normal to Siamese and from Siamese to albino.

Different visual centers in the cat receive afferents from different classes of retinal ganglion cells. The axons of ganglion cells projecting to laminae $\mathrm{A}, \mathrm{A1}$, and the $\mathrm{C}$ complex of the dLGN, RRZ, superior colliculus, pretectal complex, and ventral LGN account for all classes of ganglion cells described to date (Leventhal et al., 1980). No known classes of ganglion cells appear to be spared in the attenuation of ipsilateral retinofugal projections in the albino since axons projecting to each of these visual centers were seriously affected.

The only exception to the attenuation of ipsilateral fibers involved projections to the suprachiasmatic nuclei 
where no difference was found between normal and albino cats (Fig. 1). The crossed versus uncrossed ratio of approximately 1.4:1 of retinal ganglion fibers projecting to the albino suprachiasmatic nuclei corresponds to the ratio reported for normally pigmented cats (Hendrickson et al., 1972).

The axons destined for the suprachiasmatic nucleus deviate from the rest of the retinal ganglion cell axons at a point roughly at the junction between the optic chiasm and optic tract. This is just anterior to where a severe reduction in ipsilateral fibers is evident in the albino optic tract (Fig. 1). Our observations that all other retinofugal projections are abnormal indicate that projections to the suprachiasmatic nuclei may occur ontogenically or spatially before chiasmatic misrouting. This is consistent with the notion that misrouting of the retinofugal projections may depend upon local interactions taking place in the developing optic cup and stalk (Silver and Sapiro, 1981; Strongin and Guillery, 1981).

Future studies of the retinogeniculate pathways of the albino cat will help to elucidate the mechanism by which a known genetic defect affects the development of the nervous system.

\section{References}

Bergsma, D. R., and K. S. Brown (1971) White fur, blue eyes, and deafness in the domestic cat. J. Hered. 62: 171-185.

Cooper, M. L., and J. D. Pettigrew (1979) The retinothalamic pathways in Siamese cats. J. Comp. Neurol. 187: 313-348.

Creel, D. (1971a) Visual system anomaly associated with albinism in the cat. Nature 231: 465-466.

Creel, D. (1971b) Differences of ipsilateral and contralateral visually evoked responses in the cat: Strains compared. J. Comp. Physiol. Psychol. 77: 161-165.

Creel, D. (1980) Inappropriate use of albino animals as models in research. Pharmacol. Biochem. Behav. 12: 969-977.

Creel, D., and R. A. Giolli (1976) Retinogeniculate projections in albino and ocularly hypopigmented rats. J. Comp. Neurol. 166: $445-456$.

Creel, D., and N. B. Todd (1979) Albinism in the domestic cat. Carniv. Genet. Newsl. 3: 385.

Creel, D., C. J. Witkop, Jr., and R. A. King (1974) Asymmetric visually evoked potentials in human albinos: Evidence for visual system anomalies. Invest. Ophthalmol. 13: 430-440.

Creel, D., F. E. O'Donnell, Jr., and C. J. Witkop, Jr. (1978) Visual system anomalies in human ocular albinos. Science 201: 931-933.

Guillery, R. W. (1969) An abnormal retinogeniculate projection in Siamese cats. Brain Res. 14: 739-741.

Guillery, R. W. (1974) Visual pathways in albinos. Sci. Am. 230: 44-54.

Guillery, R. W., and J. Kaas (1971) A study of normal and congenitally abnormal retinogeniculate projections in cats. J. Comp. Neurol. 143: 73-100.

Guillery, R. W., M. D. Oberdorfer, and E. H. Murphy (1979) Abnormal retino-geniculate and geniculo-cortical pathways in several genetically distinct color phases of the mink (Mus- tela vison). J. Comp. Neurol. 185: 623-656.

Guillery, R. W., T. L. Hickey, and P. D. Spear (1981) Do blueeyed white cats have normal or abnormal retinofugal pathways? Invest. Ophthalmol. Vis. Sci. 21: 27-33.

Hendrickson, A. E., and S. B. Edwards (1978) The use of axonal transport for autoradiographic tracing of pathways in the central nervous system. In Neuroanatomical Research Tech niques, R. T. Robertson, ed., pp. 242-291, Academic Press, New York.

Hendrickson, A. E., N. Wagoner, and W. M. Cowan (1972) An autoradiographic and electron microscopic study of retinohypothalamic connections. Z. Zellforsch. 135: 1-26.

Hubel, D. H., and T. N. Wiesel (1971) Aberrant visual projections in the Siamese cat. J. Physiol. (Lond.) 218: 33-62.

Kalil, R. E., S. R. Jhaveri, and W. Richards (1971) Anomalous retinal pathways in the Siamese cat: An inadequate substrate for normal binocular vision. Science 174: 302-305.

King, R. A., and C. J. Witkop, Jr. (1976) Hairbulb tyrosinase activity in oculocutaneous albinism. Nature 263: 69-71.

LaVail, J. H., R. A. Nixon, and R. L. Sidman (1978) Genetic control of retinal ganglion cell projections. J. Comp. Neurol. 182: 399-422.

Leventhal, A. G., R. W. Rodieck, and B. Dreher (1980) Morphology and central projections of different types of retinal ganglion cells in cat and Old-World monkey. Soc. Neurosci. Abstr. 6: 582.

Prota, G. (1980) Recent advances in the chemistry of melanogenesis in mammals. J. Invest. Dermatol. 75: 122-127.

Pujol, R., M. Rebillard, and G. Rebillard (1977) Primary neural disorders in the deaf white cat cochlea. Acta Otolaryngol. (Stockh.) 83: 59-64.

Sanderson, K. J. (1971) The projection of the visual field to the lateral geniculate and the medial interlaminar nuclei in the cat. J. Comp. Neurol. 143: 101-118.

Sanderson, K. J., R. W. Guillery, and R. M. Shackelford (1974) Congenitally abnormal visual pathways in mink (Mustela vision) with reduced retinal pigment. J. Comp. Neurol. 154: $225-248$.

Searle, A. (1968) Comparative Genetics of Coat Colour in Mammals, pp. 70-145, Academic Press, New York.

Shatz, C. (1977) A comparison of visual pathways in Boston and Midwestern Siamese cats. J. Comp. Neurol. 171: 205-228.

Silver, J., and J. Sapiro (1981) Axonal guidance during development of the optic nerve: The role of pigmented epithelia and other extrinsic factors. J. Comp. Neurol. 202: 521-538.

Stone, J., J. E. Campion, and J. Leicester (1978) The nasotemporal division of the retina in the Siamese cat. J. Comp. Neurol. 180: 783-798.

Strongin, A. C., and R. W. Guillery (1981) The distribution of melanin in the developing optic cup and stalk and its relation to cellular degeneration. J. Neurosci. 1: 1193-1204.

Thibos, L. N., W. R. Levick, and R. Morstyn (1980) Ocular pigmentation in white and Siamese cats. Invest. Ophthalmol. Vis. Sci. 19: 475-486.

Wise, R. P., and R. D. Lund (1976) The retina and central projections of heterochromic rats. Exp. Neurol. 51: 68-77.

Witkop, C. J., Jr., W. C. Quevedo, Jr., and T. B. Fitzpatrick (1978) Albinism. In The Metabolic Basis of Inherited Disease, J. B. Stanbury, J. B. Wyngaarden, and D. S. Fredrickson, eds., Ed. 4, pp. 283-316, McGraw-Hill, New York. 